\title{
Face Recognition New Approach Based on Gradation Contour of Face Color
}

\author{
Iping Supriana and Yovi Pratama \\ School of Electrical Engineering and Informatics, \\ Bandung Institute of Technology, INDONESIA \\ iping@informatika.org \\ yovi.pratama@students.itb.ac.id
}

\begin{abstract}
In this paper, we introduce a new technique to recognize face image based on the gradation contour of face color. The problems remain in face recognition issue is about lighting, expressions and poses. Feature based method is considered highly successful and quite economical, however this approach is very sensitive to the light, viewing angles and poses. To handle this problem, it is better to represent the face using the 3D models, although the cost is too expensive. Related to lighting, naturally people tend to recognize the face shape based on it. According to that, the authors tried a face recognition approach using contour gradation on the face color. Variables tested in this study was Threshold Contour error, X error and Size error, then those variables are tested against the image with light illumination, yaw face and variation amount of contour lines. After testing, the best recognition results generated by $85.458 \%$ for Aberdeen face database with 150 contour lines and $90 \%$ for Yale face database. The other result showed that image with different expression was still recognizable however it cannot be recognized more than 20 degrees yaw faces. It indicated that the face contour as a feature was fairly representative to be used in face recognition.
\end{abstract}

Keywords: Face recognition; Face Feature; Face Countour; Color gradation; Curve fitting

\section{Introduction}

Face recognition is one form of biometric identification [1]. In general, face recognition technique is divided into 2 approaches, appearance-based method and feature based method [2]. The earliest work on feature based method was done by Kanade [3], who extracted a vector of 16 facial features and used a simple Euclidean distance measure for matching. This work achieved a performance rate of $75 \%$. Brunelli and Poggio[4], using Kanade's approach, computed a vector of 35 geometric features from a database of 47 people (4 images per person) and reported a $90 \%$ recognition rate. Then there was Cox et al [5] used a 30-dimensional feature vector of 35 facial features that is obtained manually and reported recognition accuracy of $95 \%$ with the 685 images. Another well-known feature-based approach was the elastic bunch graph matching method proposed by Wiskott et al. [6]. Using this architecture, the recognition rate can reach $98 \%$ for the first rank and $99 \%$ for the first 10 ranks with a gallery of 250 individuals.

Feature based method was considered highly successful [7] and quite economical, however this approach was very sensitive to the light, viewing angles, poses, etc [8]. However for feature extraction and measurement, it was not enough to be relied on [5]. It has also been claimed by Brunelli and Poggio [4] that the face recognition method based on local features of the face and the geometric relationship between the feature was less effective. The major disadvantage of these approaches was the difficulty of automatic feature detection and the fact that the implementer of any of these techniques have to make arbitrary decisions about which features were important [9].

To handle the facial variations like pose, illumination etc., it was better to represent the face used the 3D models. The 3D morphable model was a strong, effective and versatile representation of human faces [7]. Blanz et al. [10] proposed this method based on contrast and 
brightness. Then Georghiades et al. [8] also performed a 3D modeling which was supplied lighting from various angles. Although the results obtained were quite satisfy, however this approach requires a very expensive cost.

On the issue of difference on lighting source, people naturally tend to recognize things based on form of what they saw. This form can be seen due to the reflection of light from the object which is then received by the eye, either directly or indirectly (seeing picture). Not all the light bounches off the object to the eye, since the object shape is uncertain. It resulted the gradation color on the object surface, then it can be considered as a representative of the object shape. Although there are differences in exposure to an object, human can still recognize it since the object shape which is not directly represented on the color gradation.

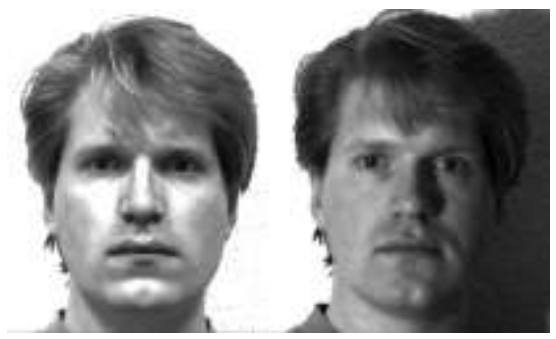

Figure 1. Different source of the light image from Yale Face Database

For example, there are 2 face images shown in Figure 1 which has the distinction of the lighting direction. This difference does not diminish the ability of human to recognize that the two faces are the same person, since there are some of the face shape that were still visible. Likewise with different facial expressions in figure 2, people can still recognize that the two faces are the same person, since the facial expressions only change the face shape in some parts of the course, while others are still recognizable.

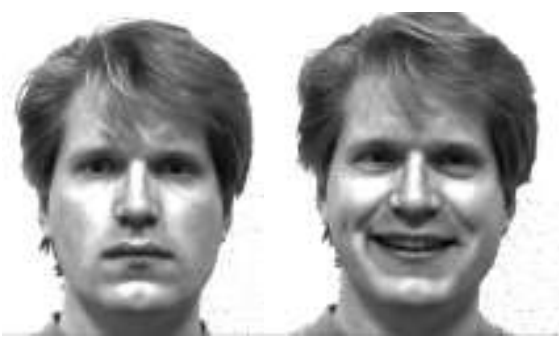

Figure 2. Different expression image from Yale Face Database

Therefore, in this paper, we described a new technique of face recognition by using contour gradation on the face color as the feature representing the face shape. These contours were extracted based on the light intensity in a grayscale channel (0-255) with the assumption of the darker intensity $(\approx 0)$ means the lower gradation of the face (based on the $\mathrm{z}$-axis) and the contrary. This technique quite economical, since the recognition was based on curve fitting used the polynomial coefficient as the model and quite strong since the use of gradation was like the 3D model.

\section{Related Works}

The earliest work on feature based method was done by Kanade [3], who extracted a vector of 16 facial features like ratios of distances, areas and angles, then used a simple Euclidean distance measure for matching. This work achieved a performance rate of $75 \%$ on a database of 20 different people with 2 images per person (one for training and one for testing). Brunelli and Poggio [4], uses Kanade's approach, computed a vector of 35 geometric features from a 
database of 47 people (4 images per person) and reported a $90 \%$ recognition rate. Figure 3 showed the geometric features used in this technique.

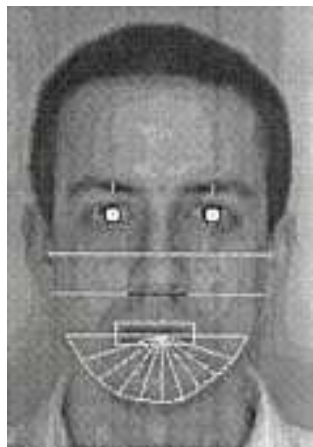

Figure 3. Geometrical features (white) used in the face recognition experiments [4]

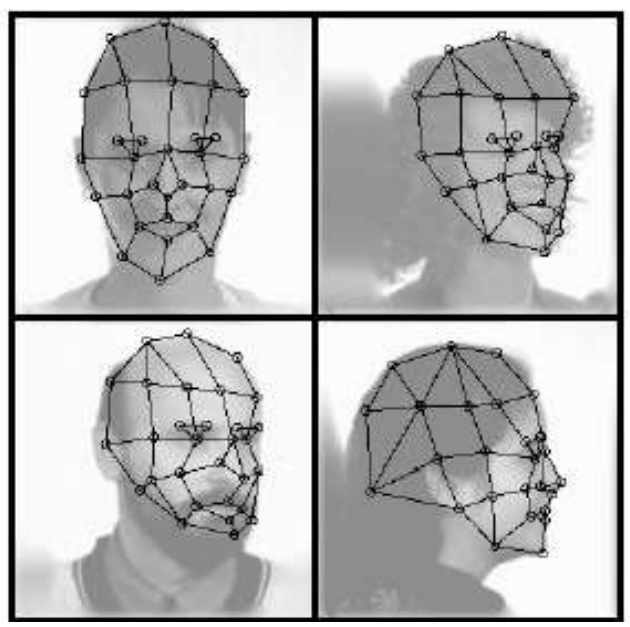

Figure 4. Grids for face recognition [6]

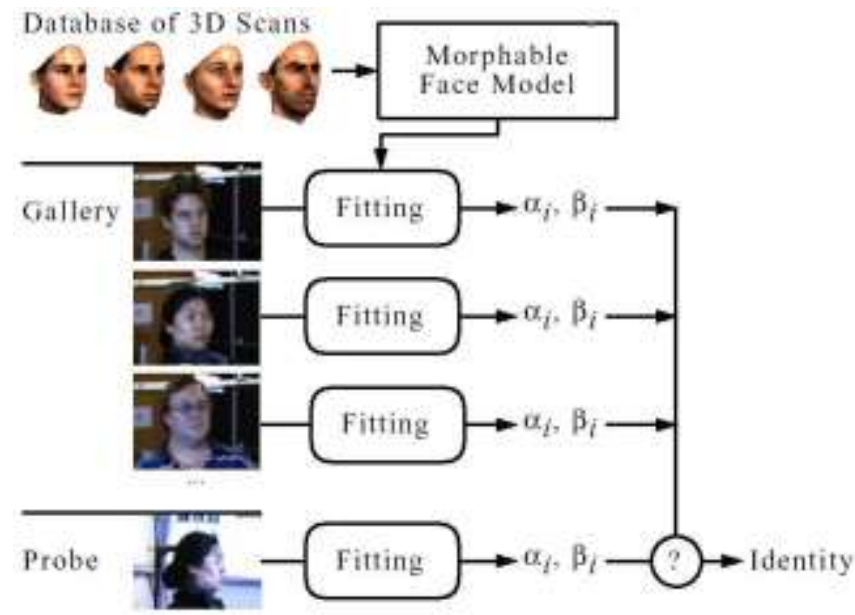

Figure 5. The Identifying scheme of 3D Morphable model [11].

Then there was Cox et al [5] used a 30-dimensional feature vector of 35 facial features that obtained manually and reported recognition accuracy of $95 \%$ with the 685 images. Another 
well-known feature-based approach was the elastic bunch graph matching method proposed by Wiskott et al. [6]. The recognition rate can reach $98 \%$ for the first rank and $99 \%$ for the first 10 ranks with a gallery of 250 individuals. The system has been enhanced to allow it to deal with different poses (Figure 4) [6] however the recognition performance on faces of the same orientation remains the same.

The other technique used is a 3D representation. Blanz et al. [10] proposed a new technique based on the 3D morphable face model that encoded shape and texture in terms of model parameters and an algorithm that recovered these parameters from 22 single image of a face. For face identification, they used the shape and texture parameters of the model that were separated from imaging parameters, such as pose and illumination. Figure 5 illustrates the scheme. To handle the extreme image variations induced by these parameters, one common approach is taken by various groups to use generative image models. For image analysis, the general strategy of all these techniques was to fit the generative model to a novel image, thereby parameterizing the novel image in terms of the model. In order to make independent identification of imaging conditions, the intrinsic model parameters of the face was separated from extrinsic imaging parameters. The separation of intrinsic and extrinsic parameters was achieved explicitly by simulating the process of image formation using 3D computer graphics technology. Although the result obtained were quite satisfy, however this approach required a very expensive cost.

The relevant studies to this research had been conducted [12] and performed face detection and face recognition in the real time. The database used in those researches is the Yale database ( 5 images as training data) and the remainder as the test images. Imagery used was normalized and the size is 60x60 pixels and the eyes position was at a specific position according to the position template. Median filtering and histogram equalization are used as preprocessing, the results was $85 \%$ when it is used subspace LDA algorithm, and it reached $82 \%$ when it is used the algorithm Kepenekci.

The other research [13] proposed a new face recognition used Line Edge Map as facial features. Then the matching process used face Hausdorf distance as its similarity measure. This study compared with the study of [14] that used same database (Yale database), the result can be seen in Table 1 . The result of the following studies $[12,13,14]$ will be compared with the result from this study that used the same face database which is the Yale face database.

Table 1. Method Comparison from [13] and [14]*.

\begin{tabular}{|c|c|}
\hline Method & Error Rate \\
\hline Edge map & $\mathbf{2 6 . 0 6 \%}$ \\
\hline Eigenface* & $24.4 \%$ \\
\hline Correlation* $^{*}$ & $23.9 \%$ \\
\hline Linear Subspace* & $21.6 \%$ \\
\hline Eigenface w/o $1^{\text {st }} 3^{*}$ & $15.3 \%$ \\
\hline LEM & $\mathbf{1 4 . 5 5 \%}$ \\
\hline Fisherface* $^{*}$ & $7.3 \%$ \\
\hline
\end{tabular}

Generally feature based approach can be summarized as in Figure 6 showing the general step of how to recognize the face based on the face feature. The first step that must be done is preparing the model that will be used to recognize the test image. The model of this approach can be obtained from the extraction process features such as the distance between facial features, contour, line edge, depth, 3D parameters, etc. This feature extraction process also applies to test images. Preprocessing also performed on the model image and test images, but it is optional. Then the last process is doing the matching feature on the test images with a model that has been prepared before in order to know the identity of the test images. 


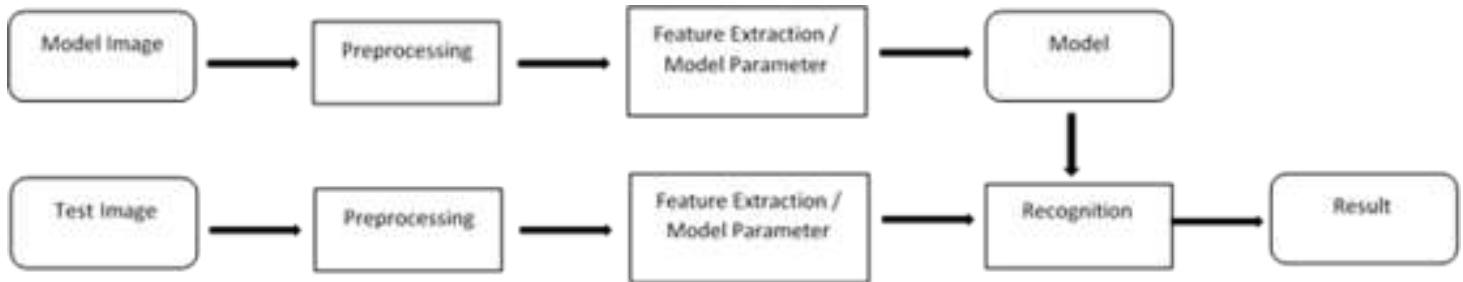

Figure 6. General Scheme of Feature Base Face Recognition

\section{Proposed Approach}

Gradation contour extraction process was done by calculating the difference of light intensity on a grayscale channel. There were 256 light intensities with the lowest value was 0 (black) and a maximum of 255 (white).

Figure 7 showed the proposed technique scheme of this study, there are 5 important steps of face recognition that we will explain more detail on the section below. There are 3 steps on the preprocessing process, which is face \& eye detection that produced both of eyes position on the image, then get the ROI of face based on golden ratio that produced the ROI of face area which the eye position will always be the same for the whole image. The Gaussian blur process is done to remove noise in the image.

The next step is to extract the face contour based on color gradation for the model image and test images. Specifically for test images, the contour is used for the feature, while the contours of the model image will be used to find the coefficients of the polynomial equation used as a model. The most recent step is to perform curve fitting to find which model is the best matches for the test images, namely by doing a search by entering points on the contour of test images into polynomial equations in the model. So that we vote the number of matching contour that has error below than treshold error, a model that has the highest number of votes is the most suitable model.

\section{A. Face \& Eye Detection}

The first process was detecting the face area (Figure 8 left), it reduced the search area for eye detection. This process controlled the cropping area of the face based on the golden ratio. The algorithm used to detect the eyes and face area were Viola Jones algorithm [15].

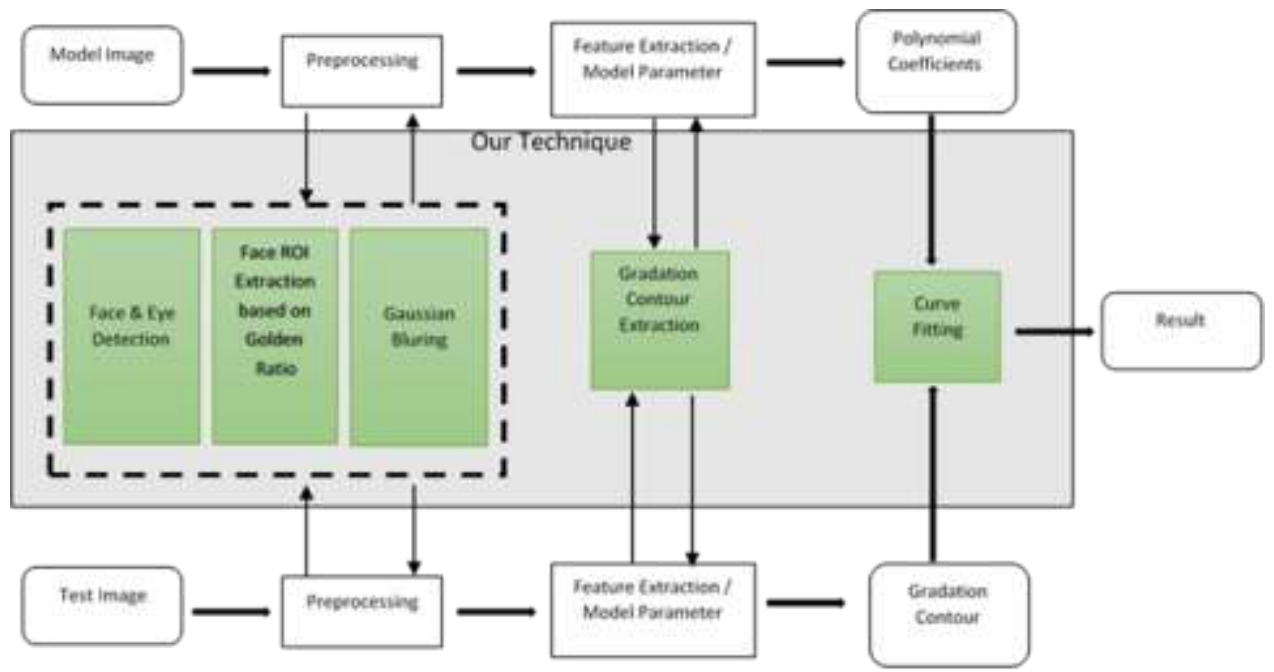

Figure 7. Scheme of Proposed Technique 
After obtaining the eye position (Figure 8 right), the next step was rotating the face in a tilted state. A tilted face can be identified by using the point of both eyes. It was done by searching for the midpoint between the two eyes as a pivot point for the rotation, then searched the tilt angle $(\theta)=\arctan (y / x)$. Having obtained the slope angle, then the image rotation is performed. Figure 9 showed the illustration of how to get the angle of yaw face image.

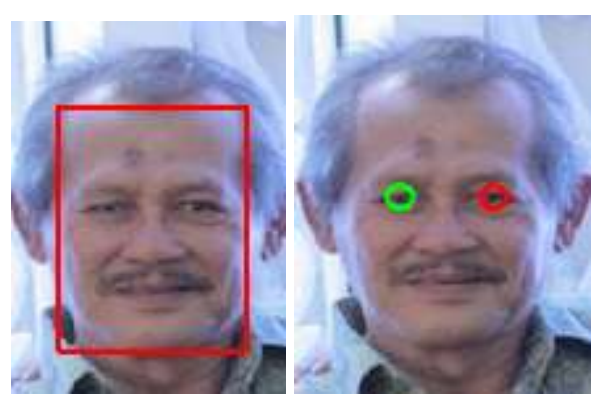

Figure 8. Face \& Eye Detection Result

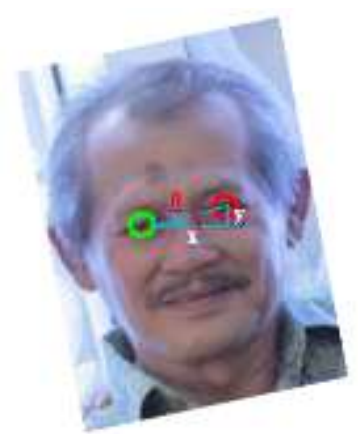

Figure 9. How to get the angle of the yaw face image based on eye location

\section{B. Face ROI Normalization based on Golden Ratio}

This process based on the eye detection, since it used the distance between the eyes as a reference to find the edge of the boundary area of the face. As reported by the http://www.phimatrix.com/face-beauty-golden-ratio/, positions can be obtained based on the distance between the eyes, among others, the position of the edge face of the right side (a), the edge position of the left side (b), the hairline (c) and the position of the chin (d) (Figure 11). The following calculations based on the Golden Ratio in constant 1.618 or 0.618 (Figure 10).

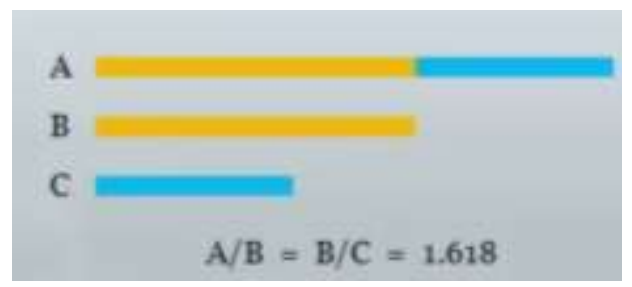

source : http://www.phimatrix.com/face-beauty-golden-ratio/

Figure 10. Golden Ratio Proportion

Xleft $=\mathrm{x}$ value of the left eye's point

Xright $=\mathrm{x}$ value of the right eye's point

Deye $=$ Xleft - Xright

Xcenter $=\mathrm{x}$ value of the center point between left \& right eye

Ycenter $=\mathrm{x}$ value of the center point between left $\&$ right eye 
$\mathrm{Xa}=((1.618 *$ Xright $)-X$ center $) / 0.618$

$\mathrm{Xb}=((1.618 * \mathrm{Xleft})-\mathrm{Xcenter}) / 0.618$

$\mathrm{Yd}=$ Ycenter $+(1.618 *$ Deye $)$

$\mathrm{Yc}=\mathrm{Yd}-(1.618 *(\mathrm{Yd}-\mathrm{Ycenter}))$

Thus, we can get 4 important points to crop the face area. Point a (Xa, Ycenter), b (Xb, Ycenter), c (Xcenter, Yc), d (Xcenter, Yd). After obtaining 4 important points, next step was cutting face area used these 4 points. To perform cutting image, there were four variables (rowstart, end row, col-start, col-end), row as the y-axis position and col as the x-axis position.

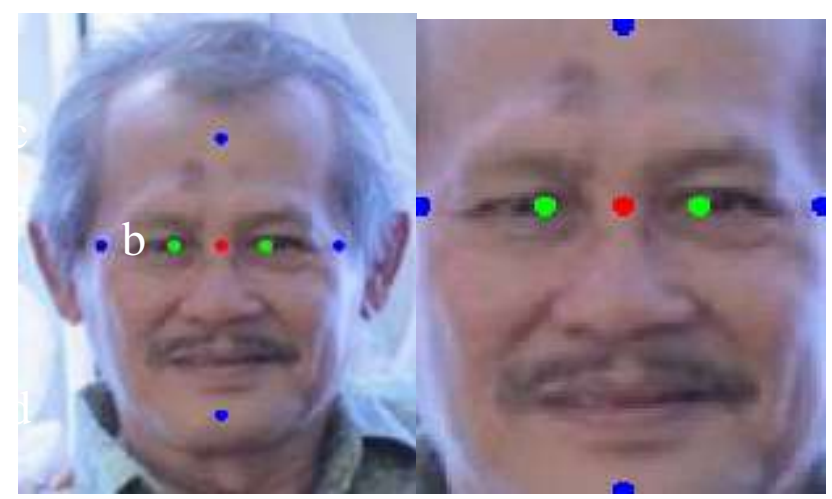

Figure 11. Face Region based on Golden Ratio

$$
\begin{aligned}
\text { row-start } & =\mathrm{Y}_{\mathrm{c}} & & \text { row-end }=\mathrm{Y}_{\mathrm{d}} \\
\text { col-start } & =\mathrm{X}_{\mathrm{a}} & & \text { col-end }=\mathrm{X}_{\mathrm{b}}
\end{aligned}
$$

Figure 11 (right) showed the result of the cutting area of the face based on the Golden Ratio. The next step was resizing the image into 250x300 pixels, it made the image ratio be the same. This technique generated result that the eye position is always be the same for the whole image (model and test image) therefore we can compare the test image and the model image with the same ratio and the same size.

\section{Gaussian Blur}

The next technique was performing noise elimination by doing the smoothing image. This study used Gaussian Blur as image smoothing technique, since according to [16] this technique is considered as the optimal technique to perform image smoothing. Gaussian smoothing operator was optimal to use in image processing. Gaussian function $\mathrm{g}$ in the coordinates $\mathrm{x}, \mathrm{y}$ depended on the value of the variance $\sigma^{2}$.

$$
g(x, y, \sigma)=\frac{1}{2 \pi \sigma^{2}} e^{-\left(\frac{x^{2}+y^{2}}{2 \sigma^{2}}\right)}
$$

\section{Contour Extraction based on Color Gradation}

Techniques to perform contour extraction were utilizing colors gradation contained in each pixel of the image. Gradation was taken by the level of grayscale color, since the imagery used in this study was a color image, then it was necessary to convert the color image to the RGB images. The techniques are:

$$
\operatorname{Gray}_{\mathrm{ij}}=\left(\mathrm{R}_{\mathrm{ij}}+\mathrm{G}_{\mathrm{ij}}+\mathrm{B}_{\mathrm{ij}}\right) / 3,(\mathrm{i}=0,1,2, \ldots, \mathrm{m}),(\mathrm{j}=0,1,2, \ldots, \mathrm{n})
$$


$R_{i j}$ is the red value on pixel (i, j), $G_{i j}$ is the green value on pixel (i,j), $B_{i j}$ is the blue value on pixel $(i, j), m$ is the width and $n$ is the height of the image. Figure 12 shows the example of 2 Line image pixels on grayscale channel.

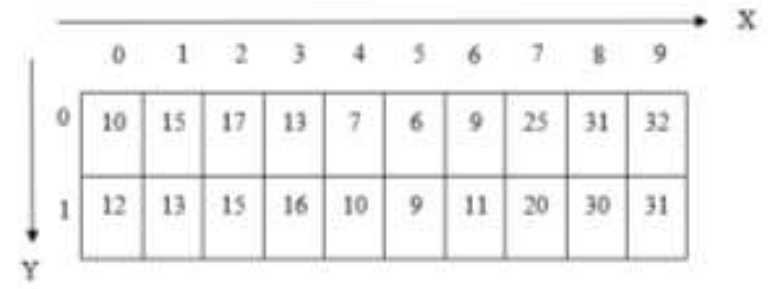

Figure 12. Example of Two Lines image pixels grayscale channel.

In general, two dimensional digital images have two coordinate axes, namely $\mathrm{X}$ and $\mathrm{Y}$, where $\mathrm{X}$ for horizontal and $\mathrm{Y}$ for vertical. It needs $\mathrm{Z}$ axis to get 3 dimensional coordinates, while to get the contour we use the $\mathrm{X}$-axis and $\mathrm{Z}$-axis. We can get the $\mathrm{Z}$-axis value by the grayscale value on each pixel of the image. See Figure 13 showing the visualization contour of Figure 12.

The next technique was dividing one contour line into several lines based on changes of the direction. As illustrated in Figure 14, the contour as the image is divided into several parts. The purpose of dividing it was to facilitate the search for the coefficients of quadratic equations of the contour line. Set of contours (Figure 15) was used as the face feature.

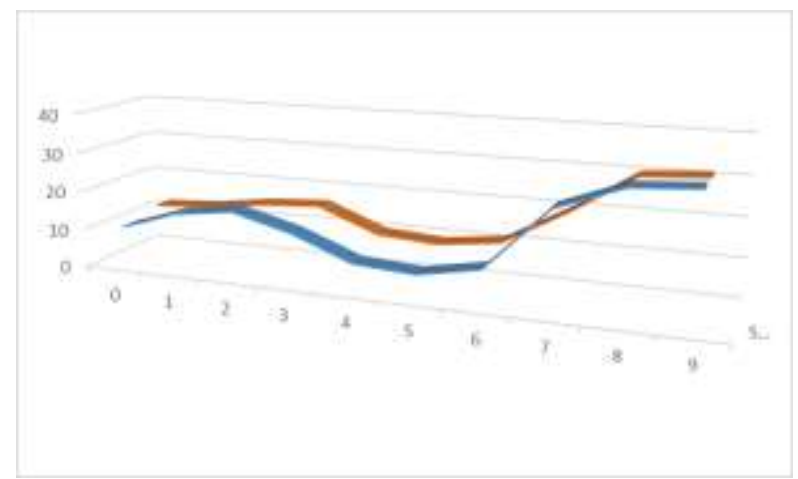

Figure 13. Contour Visualization. The grayscale value on each pixel of the image

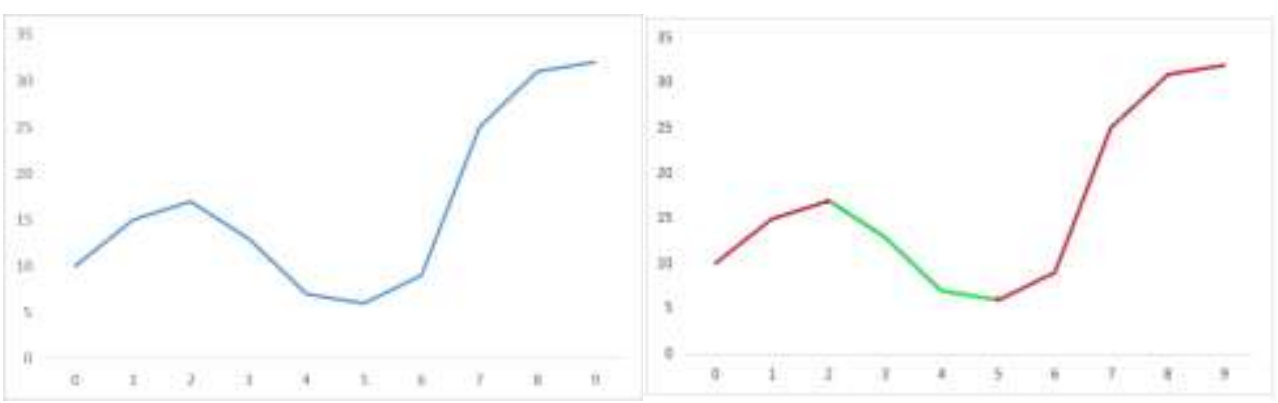

Figure 14. The Contour that is divided into Up and Down part. 


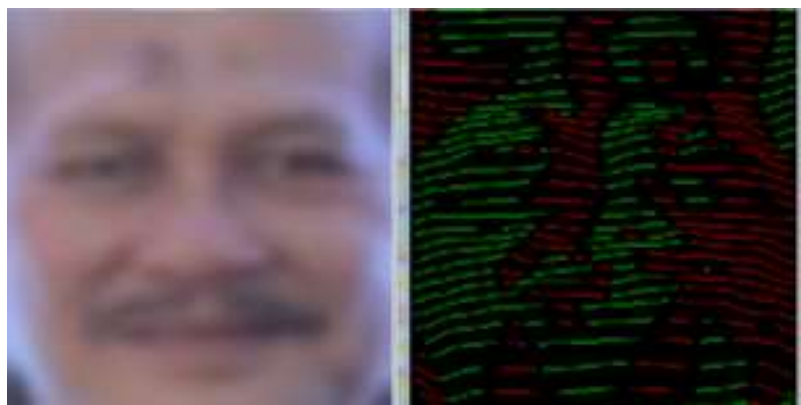

Figure 15. Face Contour Example

\section{E. Face Recognition based on curve fitting}

To check whether the two faces are the same or not, it had been performed a comparison between the contours that has been extracted in the previous technique that was by doing Curve Fitting between the contours with other contours. Consider the following example with 2 contours (Table 2).

Table 2. Contour $1 \&$ Contour 2

\begin{tabular}{|l|l|l|l|}
\hline $\mathrm{x}$ & 0 & 1 & 2 \\
\hline $\mathrm{y}$ & 10 & 15 & 17 \\
\hline
\end{tabular}$\quad$\begin{tabular}{|l|l|l|l|}
\hline $\mathrm{x}$ & 0 & 1 & 2 \\
\hline $\mathrm{y}$ & 8 & 14 & 16 \\
\hline
\end{tabular}

The first step was finding the polynomial coefficients of the contour 1 , the order of polynomial used in the study is 1 to 20 . However, the search stops if an error has been reached 0 . Before searching the coefficient, the second position of contour should be normalized. Likewise with the point 0 , the whole point ( $\mathrm{x}$ and $\mathrm{y}$ ) was reduced by the first point ( $\mathrm{x}$ and $\mathrm{y}$ ).

$$
\mathrm{X}_{\mathrm{i}}=\mathrm{X}_{\mathrm{i}}-\mathrm{X}_{1,} \quad \mathrm{Y}_{\mathrm{i}}=\mathrm{Y}_{\mathrm{i}}-\mathrm{Y}_{1,} .
$$

$\begin{array}{lll}\text { Contour } 1 \rightarrow & \mathrm{X}=(0,1,2) & \rightarrow \mathrm{X}=(0,1,2) \\ & \mathrm{Y}=(10,15,17) & \rightarrow \mathrm{Y}=(0,5,7) \\ \text { Contour } 2 \rightarrow & \mathrm{X}=(0,1,2) & \rightarrow \mathrm{X}=(0,1,2) \\ & \mathrm{Y}=(8,14,16) & \rightarrow \mathrm{Y}=(0,6,8)\end{array}$

Contour 1 searching coefficient as follows:

$\mathrm{X}=(0,1,2)$

$\mathrm{Y}=(0,5,7)$

$1^{\text {st }}$ Order $\rightarrow 3.5 \mathrm{x}+0.5$

$2^{\text {nd }}$ Order $\rightarrow-1.5 \mathrm{x}^{2}+6.5 \mathrm{x}+-7.39 \mathrm{E}-32$

err $=0.6661$

$3^{\text {rd }}$ Order $\rightarrow-0.5 \mathrm{x}^{3}+0.0 \mathrm{x}^{2}+5.5 \mathrm{x}+-2.95 \mathrm{E}-31$

err $=2.46 \mathrm{E}-32$

$4^{\text {th }}$ Order $\rightarrow-0.214 \mathrm{x}^{4}+0.0 \mathrm{x}^{3}+0.0 \mathrm{x}^{2}+5.21 \mathrm{x}+0.0$

err $=9.86 \mathrm{E}-32$

err $=0.0$

Thus, most polynomial fit to the contour 1 was

$$
-0.214 \mathrm{x}^{4}+0.0 \mathrm{x}^{3}+0.0 \mathrm{x}^{2}+5.21 \mathrm{x}+0.0 .
$$

The next step to prove the equation between contour 2 and contour 1 is entering the $\mathrm{X}$ value of the contour 2 into the polynomial equation of contour 1 . 
Contour 2

$\mathrm{X}=(0,1,2)$

$\mathrm{Y}=(0,6,8)$

$\mathrm{f}(\mathrm{X}), \mathrm{X}=0 \rightarrow-0.214(0)^{4}+0.0(0)^{3}+0.0(0)^{2}+5.21(0)+0.0=0$

$\mathrm{f}(\mathrm{X}), \mathrm{X}=1 \rightarrow-0.214(1)^{4}+0.0(1)^{3}+0.0(1)^{2}+5.21(1)+0.0=4.9996$

$\mathrm{f}(\mathrm{X}), \mathrm{X}=2 \rightarrow-0.214(2)^{4}+0.0(2)^{3}+0.0(2)^{2}+5.21(2)+0.0=8.708$;

Then looking for errors using the least square error equation below:

$$
\text { err }=\frac{\sum_{i=1}^{n}\left|y_{i}-f\left(x_{i}\right)\right|}{n}
$$

err $=(|0-0|+|6-4.9996|+|8-8.708|) / 3=0.0974$

These steps were performed on all contours which successfully extracted, then voted on all contours that have error below the error threshold.

\section{Result and Evaluation}

There were 2 face databases used in this study, the face image downloaded from the link http://pics.stir.ac.uk/zips/Aberdeen.zip, such as color images of 90 individuals ranging from 1 to 18 images per individual. There were some variations in lighting, 8 variations of view point with varying resolution between $336 \times 480$ to $624 \times 544$ and Yale database downloaded from the link http://vision.ucsd.edu/content/yale-face-database, such as grayscale images of 15 individuals with 11 images per individual with each such variation, variations of the lighting direction (left, right, middle), variations of expressions (happy, sad, surprised, wink), variations of accessories (glasses, non-glasses).

The separation of test images and model image with proportions, one image of the model and the remainder are used as test images. Selection of the model image was done by cross validation method, by taking the highest first rank of cross validation results.

For a number of the contour line 50, the results of the highest accuracy in Aberdeen face database was $\mathbf{8 4 . 9 1 9} \%$. Where it has Size error $=10$, Contour Error $=9$, and $\mathrm{X}$ error $=8$ (Table 3 ). While the accuracy of the First 10 Rank with those 3 variables reached $\mathbf{9 5 . 5 1 2} \%$. While at Yale database, it resulted the highest accuracy of $\mathbf{9 0 \%}$ with a size error $=5$, Contour Error $=$ 44-46 with $X$ error $=8$ and Contour Error $=40-50$ with $X$ error $=9$ (Table 4). Then the accuracy of the First 10 Rank with those 3 variables reached $\mathbf{9 8 . 6 6 7 \%}$ with the same number of contour lines.

Table 3. Result on Aberdeen Face Database (50 line contour)

\begin{tabular}{|c|c|c|c|c|c|c|c|}
\hline \multicolumn{8}{|c|}{ Size error $=10$} \\
\hline $\mathbf{X}$ & \multicolumn{7}{|c|}{ Kontur Error } \\
\hline \multirow{2}{*}{$\begin{array}{l}\text { Error } \\
7\end{array}$} & 8 & 9 & 10 & 11 & 12 & 13 & 14 \\
\hline & 83.124 & 82.944 & 82.585 & 82.585 & 83.303 & 83.124 & 82.944 \\
\hline 8 & 84.201 & 84.919 & 84.56 & 84.56 & 84.022 & 83.842 & 84.022 \\
\hline 9 & 84.56 & 84.022 & 84.201 & 84.022 & 84.381 & 83.662 & 83.842 \\
\hline 10 & 84.74 & 84.56 & 84.381 & 83.662 & 83.483 & 82.944 & 83.842 \\
\hline 11 & 83.483 & 83.124 & 82.585 & 83.124 & 83.124 & 82.406 & 83.124 \\
\hline 12 & 82.944 & 83.483 & 83.483 & 82.765 & 82.226 & 82.406 & 82.765 \\
\hline
\end{tabular}


Table 4. Result on Aberdeen Face Database (50 line contour)

\begin{tabular}{|l|lllllll|}
\multicolumn{2}{l}{$\begin{array}{l}\text { Size error }=\mathbf{5} \\
\mathbf{X}\end{array}$} & \multicolumn{1}{l}{ Kontur Error } \\
Error & $\mathbf{4 2}$ & $\mathbf{4 3}$ & $\mathbf{4 4}$ & $\mathbf{4 5}$ & $\mathbf{4 6}$ & $\mathbf{4 7}$ & $\mathbf{4 8}$ \\
$\mathbf{7}$ & 84 & 84 & 84 & 84 & 84 & 84 & 84 \\
\hline 8 & 89.333 & 89.333 & 90 & 90 & 90 & 89.333 & 89.333 \\
9 & 90 & 90 & 90 & 90 & 90 & 90 & 90 \\
\hline 10 & 89.333 & 89.333 & 89.333 & 89.333 & 89.333 & 89.333 & 89.333 \\
\hline 11 & 88.667 & 88.667 & 88.667 & 88.667 & 88.667 & 88 & 88 \\
\hline 12 & 88.667 & 88.667 & 88.667 & 89.333 & 89.333 & 89.333 & 89.333 \\
\hline
\end{tabular}

For a number of contour lines of the highest yields, the best accuracy of the testing Aberdeen face database was 150 contour lines with an accuracy reached $\mathbf{8 5 . 4 5 8} \%$. Average execution time per test images is 1.06 seconds. Table 5 showed all results of experiment on different number of contour lines and Figure 16 showed the effect against accuracy, while Figure 17 showed the effect against execution time.

From the test results, failed face recognition is caused by the difference of light source between models and test images, where the failed test image was the number of 13-15, and number 4 for the image of the 4 pieces per individual. It proved that illumination of lighting created facial contours that are formed so differently.

Table 5. The Result on Aberdeen Face Database on different number of line contour, A is the number of line contour, B is the accuracy, C, D, E is execution time

\begin{tabular}{|l|l|l|l|l|}
\hline $\mathbf{A}$ & $\mathbf{B}(\boldsymbol{\%})$ & $\mathbf{C}(\mathbf{m s})$ & $\mathbf{D}(\mathbf{m s})$ & $\mathbf{E}(\mathbf{s})$ \\
\hline 20 & 75.404 & 43819 & 78.66965889 & 0.078669659 \\
\hline 30 & 81.149 & 55399 & 99.45960503 & 0.099459605 \\
\hline 50 & 84.919 & 128618 & 230.9120287 & 0.230912029 \\
\hline 100 & 85.278 & 397131 & 712.9820467 & 0.712982047 \\
\hline $\mathbf{1 5 0}$ & $\mathbf{8 5 . 4 5 8}$ & $\mathbf{5 9 1 8 7 9}$ & $\mathbf{1 0 6 2 . 6 1 9 3 9}$ & $\mathbf{1 . 0 6 2 6 1 9 3 9}$ \\
\hline 300 & 84.919 & 769592 & 1381.67325 & 1.38167325 \\
\hline
\end{tabular}

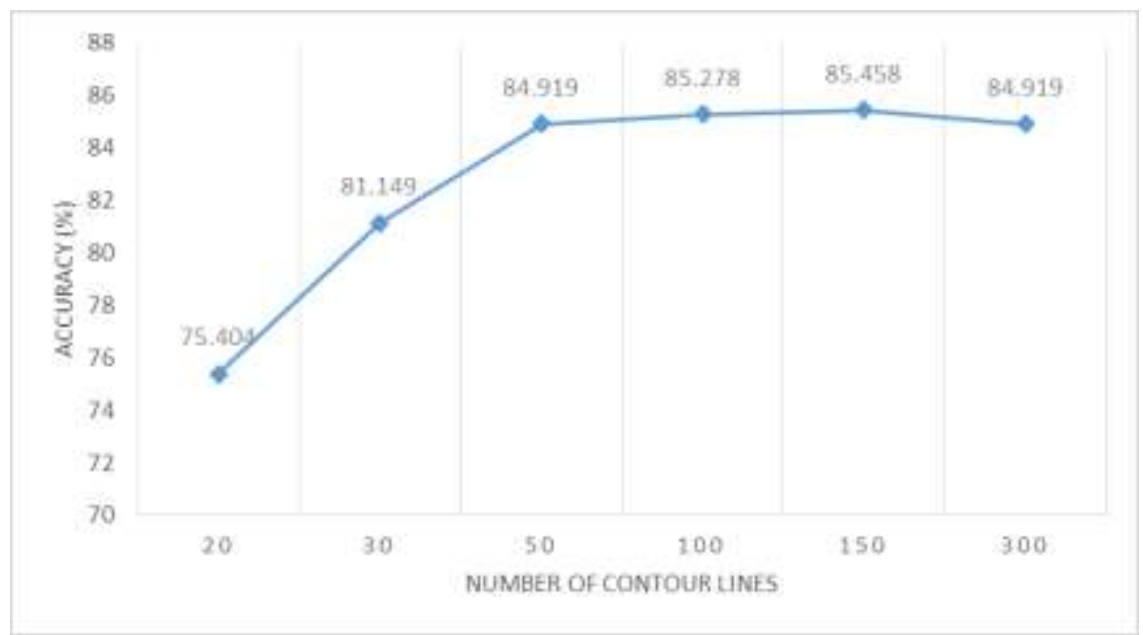

Figure 16. Number Effect of contour lines against total accuracy (\%). 


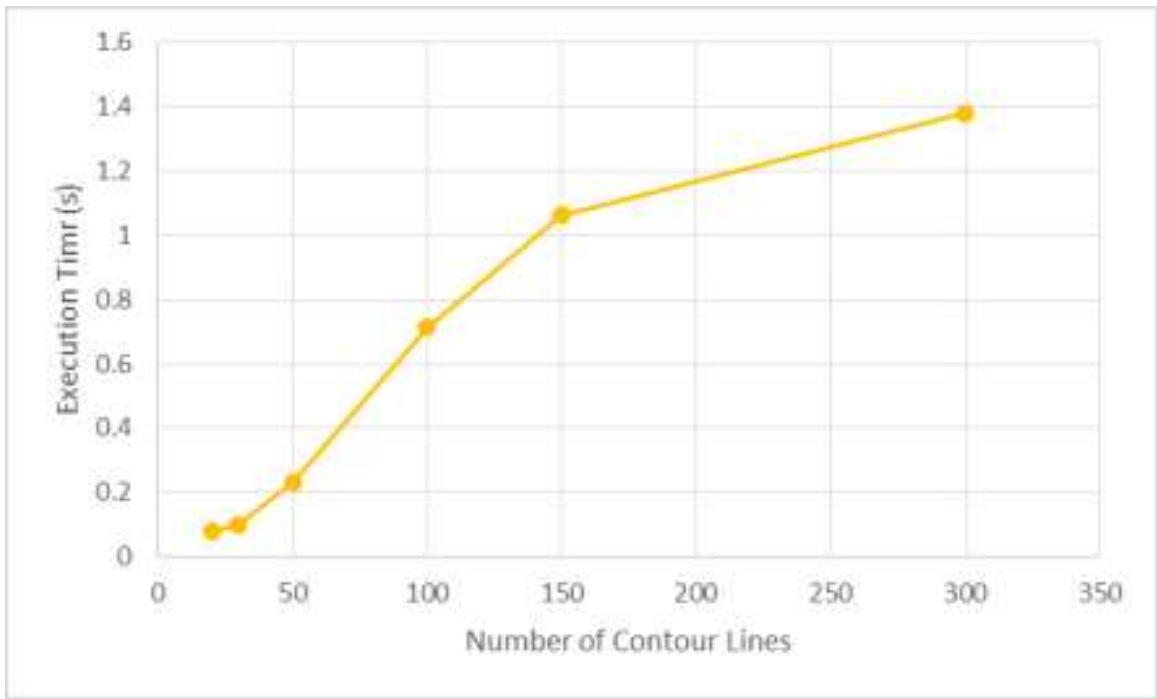

Figure 17. Number effect of contour lines against total execution time (s).

The same problem occurred in Yale face database. The lighting direction affected the success of recognition, however to case different expression, facial contour approach still showed good results.

The recognition of the $22^{0}$ yaw face resulted the top 5 ranks with a similarity score below $50 \%$. In addition, the recognition of the $45^{\circ}$ yaw face resulted the top 5 ranks, with a score below $20 \%$ similarity.

From the related work section we have studied the relevant technique with this study that are used the same face database, which is Yale face database. There are many techniques used from the following studies $[12,13,14]$. We compare the result from their technique with our study. Table 6 showed the comparison between our approach results and the results of face recognition method proposed by the previous study by using the Yale face database.

Table 6. Comparison between proposed techniques with the other method

\begin{tabular}{|c|c|}
\hline Metode & Accuration \\
\hline Fisherface [14] & $92.70 \%$ \\
\hline $\begin{array}{l}\text { Face Color Gradation } \\
\text { Approach }\end{array}$ & $90.00 \%$ \\
\hline Line Edge Map [13] & $85.45 \%$ \\
\hline Subspace LDA [12] & $85 \%$ \\
\hline Eigen Face w/o $1^{\text {st }} 3$ [14] & $84.70 \%$ \\
\hline Kepenekci [12] & $82 \%$ \\
\hline Linear Subspace [14] & $78.40 \%$ \\
\hline Correlation [14] & $76.10 \%$ \\
\hline Eigen Face [14] & $75.60 \%$ \\
\hline Edge Map [14] & $73.94 \%$ \\
\hline
\end{tabular}

\section{Conclusion}

Based on the result, it can be concluded that the difference of Illumination still affects the success of proposed method, where most of errors indicated by the Aberdeen with an average score are below 50\%. The differences of the lighting direction also quite affected the success of the recognition, however most of them were still quite good, where the majority indicated by the test images at Yale database were still recognizable, with the notes, the similarity score between model and test image was below $50 \%$. 
Then the difference of expression did not diminish the success of the recognition, from the test results, only a small percentage of failure was caused by different expression. This approach only performed facial recognition with a tolerance of yaw face under $20^{\circ}$.

Based on tested accuracy obtained, the face recognition method with color gradations contour approach was quite representative as a unique feature of a face.

In the next futures work will be explored : (1) The number of optimum contour that needs to be calculated and (2) the best conditions of poses that can be processed satisfactorily with our approach

\section{References}

[1]. S. T. Gandhe, K. T. Talele, and a G. Keskar, "Face Recognition Using Contour Matching," Int. J., no. May, 2008

[2]. N. B. Boodoo-Jahangeer and S. Baichoo, "Face Recognition Using Chain Codes," J. Signal Inf. Process., vol. 04, no. 03, pp. 154-157, 2013

[3]. T. Kanade, "Picture Processing System by Computer Complex and Recognition of Human Faces," doctoral dissertation, Kyoto University. 1973

[4]. R. Brunelli and T. Poggio, "Face recognition: features versus templates," IEEE Trans. Pattern Anal. Mach. Intell., vol. 15, no. 10, pp. 1042-1052, 1993

[5]. I. J. Cox and P. N. Yianilos, "Feature-Based Face Recognition Using Mixture Distance," no. October, 1995

[6]. L. Wiskott, J. Fellous, N. Kruger, and C. Von Der Malsburg, "Face Recognition by Elastic Bunch Graph Matching," IEEE Trans. Pattern Anal. Mach. Intell., vol. 19, no. 7, pp. 1-23, 1996

[7]. G. Ramkumar and M. Manikandan, "FACE RECOGNITION - SURVEY," Int. J. Adv. Sci. Technol., pp. 260-268, 2013

[8]. A. S. Georghiades, P. N. Belhumeur, and D. J. Kriegman, "From few to many: Illumination cone models for face recognition under variable lighting and pose," IEEE Trans. Pattern Anal. Mach. Intell., vol. 23, no. 6, pp. 643-660, 2001

[9]. R. Cendrillon, "Real Time Face Recognition using Eigenfaces," Eff. Br. mindfulness Interv. acute pain Exp. An Exam. Individ. Differ., vol. 1, pp. 1689-1699, 1999

[10]. V. Blanz and T. Vetter, "Face recognition based on fitting a 3D morphable model," IEEE Trans. Pattern Anal. Mach. Intell., vol. 25, no. 9, pp. 1063-1074, 2003

[11]. X. Lu, X. Lu, and E. Lansing, "Image analysis for face recognition," Pers. Notes, May, vol. 5, pp. 1-37, 2003

[12]. A. Dutta, B. Shrestha, and L. Kansakar, "Real Time Face Tracking and Recognition (RTFTR)," 2009

[13]. Y. S. Gao and M. K. H. Leung, "Face recognition using line edge map," IEEE Trans. Pattern Anal. Mach. Intell., vol. 24, no. 6, pp. 764-779, 2002

[14]. P. N. Belhumeur, J. P. Hespanha, and D. J. Kriegman, "Eigenfaces vs. fisherfaces: Recognition using class specific linear projection," IEEE Trans. Pattern Anal. Mach. Intell., vol. 19, no. 7, pp. 711-720, 1997

[15]. P. Viola and M. Jones, "Rapid object detection using a boosted cascade of simple features," Comput. Vis. Pattern Recognit., vol. 1, pp. I-511-I-518, 2001

[16]. M. S. Nixon and A. S. Aguado, Feature Extraction \& Image Processing, Second Edi. London: Elsevier, 2008 


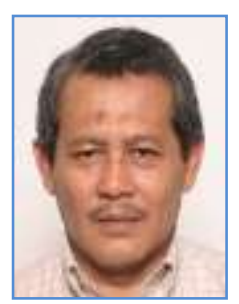

Iping Supriana received the docteur-ingénieur degree in Informatics from the Institute National Polytechnique, Grenoble, French in 1985. He is currently a Professor of the School of Electrical and Informatic Engineering, Bandung Institute of Technology, Indonesia. His research interests include: information automation, dynamic computer graphic, image analysis, recognition system, and image interpretation. He has authored or coauthored over 50 published articles. He is the inventor and the implementer of the Digital Mark Reader (DMR). DMR is software employed for evaluating examination results automatically using computer scanning. DMR iswidely used in Indonesian education institution. He is also the inventor and the implementer of the Digital Scan Meter (DSM). DSM is software employed for reading and reporting the electric power consumption. The software convert the image photo contains numbers into the digital format for billing requirement. DSM is widely in used in the National Electrical Company in West Java, Indonesia.

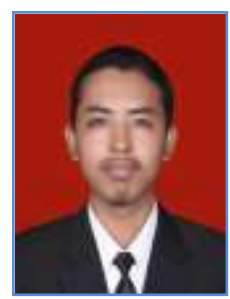

Yovi Pratama is currently a student of Magister of Informatics at Bandung Institute of Technology, Indonesia. He is working on his thesis. His research interests include digital image processing and pattern recognition. He had completed his bachelor program at computer science faculty at Sriwijaya University in 2012. 